\title{
Enzyme-linked immunosorbent assay of nicotine metabolites
}

\author{
Akiko Matsumoto $\cdot$ Toshihiro Ino $\cdot$ Mitsuhiro Ohta $\cdot$ \\ Tetsuya Otani $\cdot$ Sachiko Hanada $\cdot$ Atsushi Sakuraoka \\ Akane Matsumoto $\cdot$ Masayoshi Ichiba $\cdot$ Megumi Hara
}

Received: 16 September 2009/Accepted: 13 December 2009/Published online: 8 January 2010

(C) The Japanese Society for Hygiene 2010

\begin{abstract}
Introduction The level of cotinine in biological specimens, such as serum, urine, and saliva, measured by gas or liquid chromatography is the most validated and reliable indicator of exposure to tobacco smoke. However, chromatographic methods are not always suitable for all types of situations.
\end{abstract}

Electronic supplementary material The online version of this article (doi:10.1007/s12199-009-0129-2) contains supplementary material, which is available to authorized users.

\author{
A. Matsumoto $(\bowtie)$ \\ Department of Pharmaceutical Science, School of \\ Pharmacology, Colorado University, Aurora, CO 80045, USA \\ e-mail: Akiko.Matsumoto@ucdenver.edu; \\ matsumoa@cc.saga-u.ac.jp \\ T. Ino \\ Ino Clinic, Saitama 360-0014, Japan \\ T. Ino \\ Department of Nursing, Gunma Paz College, \\ Gunma 370-0044, Japan \\ M. Ohta \\ Department of Medical Biochemistry, \\ Kobe Pharmaceutical University, Kobe 658-8558, Japan \\ T. Otani \\ Department of Health Policy, National Research Institute \\ for Child Health and Development, Tokyo 157-8535, Japan \\ S. Hanada $\cdot$ A. Sakuraoka \\ Cosmic Corporations, Tokyo 112-0002, Japan \\ A. Matsumoto $\cdot$ M. Ichiba $\cdot$ M. Hara \\ Department of Social Medicine, Saga Medical School, \\ Saga 849-8501, Japan
}

Methods We validated a commercially available enzymelinked immunosorbent assay (ELISA) that uses a polyclonal antibody to cotinine as a practical alternative to chromatographic methods.

Results The cotinine antibody cross-reacts to 3-hydroxycotinine (3HC) and its glucuronide, thus generating a value for immunoreactive (IR) cotinine, which is a complex comprising cotinine, $3 \mathrm{HC}$, and $3 \mathrm{HC}$-glucuronide. The levels of IR cotinine in the urine of kindergarten children closely correlated with those of cotinine measured by gas chromatography-mass spectrometry (GC-MS) and reflected the smoking behavior of their parents more precisely than cotinine levels determined by GC-MS.

Discussion Our findings showed that the cotinine-based ELISA can be a practical biomarker of exposure to tobacco smoke.

Keywords Cotinine - ELISA - Environmental tobacco smoke $\cdot 3$-Hydroxycotinine

\section{Introduction}

Cotinine concentrations in biological samples have been measured by gas (GC) and liquid (LC) chromatography to monitor exposure to tobacco smoke [1-3]. Analytical methods should be useful under many circumstances, such as when parents entering smoking cessation programs to parents request a health check for a child or when families present at a pediatric unit with a sick child.

However, GC and LC are not always suitable for all types of situations because both of these methods require expensive equipment, skilled operators, and large amounts of samples [1, 4-7]. In addition, both GC and MS are lengthy procedures, which is a particular disadvantage for 
high-throughput screening. Therefore, alternative analytical procedures, such as the enzyme-linked immunosorbent assay (ELISA), may be useful when large numbers of samples need to be analyzed rapidly or when suitable chromatographic equipment is unavailable. A number of reports have already suggested the advantages of immunoassay systems [6-9], however, relatively little information is available on such systems in terms of, for example, cross-reactivity or their merits and disadvantages. Here, we have validated a commercially available competitive ELISA as a biological monitor of exposure to tobacco smoke using 113 urine specimens from children. We have also compared the findings to those of GC-MS and highperformance liquid chromatography (HPLC).

\section{Method}

\section{ELISA assay}

We validated a competitive ELISA kit (Cosmic Corp, Tokyo, Japan) using 96-well plates that had been coated with a rabbit anti-cotinine-4-bovine- $\gamma$-globulin polyclonal antibody. In brief, the plates were first incubated with $1 \%$ bovine serum albumin (BSA) and $5 \mu \mathrm{L}$ of urine samples, followed by the addition of $100 \mu \mathrm{L}$ horseradish peroxidaselabeled cotinine. The mixture was then incubated at $20-25^{\circ} \mathrm{C}$ for $1 \mathrm{~h}$. After three washes with $1 \% \mathrm{BSA}$, the peroxidase substrate, tetramethylbenzidine, and $\mathrm{H}_{2} \mathrm{O}_{2}$ (Kirkegaad \& Perry Laboratories, Gaithersburg, MD) were added and the mixture incubated for $30 \mathrm{~min}$. Phosphoric acid was then added to the wells to stop the enzyme reaction. Enzyme activity was detected at $450 \mathrm{~nm}$ using an ELISA reader ( $\mathrm{E}_{\max }$; Molecular Devices, Sunnyvale, CA). Immunoreactive (IR) cotinine concentrations were calculated from a standard curve. Standard urine samples were prepared by spiking blank urine (urine from an infant residing in the countryside with non-smokers) with a series of cotinine dilutions. The cotinine level in the blank urine determined by GC-MS was $6 \mathrm{ng} / \mathrm{mL}$, and those of cotinine, 3-hydroxycotinine (3HC), and 3HC-glucuronide determined by HPLC were $<22,73$, and $<20 \mathrm{ng} / \mathrm{mL}$, respectively. Details of the GC-MS and HPLC analyses are described below.

\section{Evaluation of cross-reactivity}

We evaluated the cross-reactivity of the ELISA to the major nicotine metabolites, namely, nicotine, nicotine glucuronide, cotinine glucuronide, 3HC, and 3HC-glucuronide. The results of this evaluation are given in Table 1. Standard nicotine solutions were purchased from Wako Pure Chemical Industries (Osaka, Japan), and nicotine- $N$ - $\beta$-glucuronide, cotinine- $N$ - $\alpha$-D-glucuronide, $\left(3^{\prime} \mathrm{R}, 5^{\prime} \mathrm{S}\right)-3^{\prime}$-hydroxycotinine, and trans-3'-hydroxycotinine $O-\beta$-D-glucuronide were purchased from Toronto Research Chemicals (North York, ON, Canada). Standard solutions were diluted in methanol and then further diluted in $1 \% \mathrm{BSA}$; the reaction solution included methanol (up to 0.2 in $50 \%$ effective dose).

\section{Study participants}

Ninety-three urine specimens were collected from 93 Japanese children aged 3 to 6 years in order to evaluate second-hand tobacco smoke exposure of these children. All of the children were attending a kindergarten and had undergone a medical examination. To obtain information on the smoking habits of the parents, we asked the parents to complete a self-administered questionnaire at the start of the study. Urine samples collected from 19 otherwise healthy smokers who consulted a physician regarding strategies to quit smoking were stored at $-20^{\circ} \mathrm{C}$ until analyzed by ELISA, HPLC, and GC-MS. The adult donors and the parents of the kindergarten children provided written informed consent to all procedures associated with the study, and the urine samples and questionnaire were assigned ID numbers to protect the identities of the participants. The study design was approved by the Medical Ethics Committee of Saga Medical School.

Table 1 Evaluation of cross-reactivity with nicotine metabolites

\begin{tabular}{llcrr}
\hline Major nicotine metabolites & Molecular weight & $\mathrm{ED}_{50}(\mathrm{ng} / \mathrm{mL})$ & $\mathrm{ED}_{50}(\mathrm{M})$ & Cross-reactive ratio $^{\mathrm{a}}(\%)$ \\
\hline Cotinine & 176.22 & 5.2 & $2.95 \times 10^{-8}$ & 100.0 \\
Nicotine & 162.23 & 2140.8 & $1.32 \times 10^{-5}$ & 0.2 \\
Nicotine glucuronide & 338.36 & 37919.8 & $1.12 \times 10^{-4}$ & 0.0 \\
Cotinine glucuronide & 352.34 & 3764.2 & $1.07 \times 10^{-5}$ & 0.3 \\
3'-Hydroxycotinine & 192.21 & 5.6 & $2.91 \times 10^{-8}$ & 101.3 \\
3'-Hydroxycotinineglucuronide & 368.34 & 4.9 & $1.33 \times 10^{-8}$ & 221.8 \\
\hline
\end{tabular}

$E D_{50} 50 \%$ effective dose

${ }^{\text {a }}$ Cross-reactive ratio is the ratio of $\mathrm{ED}_{50}(\mathrm{M})$ of each metabolite to that of cotinine (M) 
GC-MS analysis

Urinary cotinine levels (free cotinine) and total levels of cotinine and cotinine glucuronide (total cotinine) were determined. Glucuronide conjugates were prepared as described by Benowitz et al. [10]. Total and free cotinine was measured with and without hydrolysis, respectively. Conjugates in urine samples were hydrolyzed by adding sodium hydroxide to a final concentration of $1.14 \mathrm{M}$ and then incubating the product at $75^{\circ} \mathrm{C}$ for $1 \mathrm{~h}$. Cotinine was extracted and determined using a modification of the method of Man et al. [4]. Briefly, $0.45 \mathrm{~mL}$ of urine was placed in $1.5-\mathrm{mL}$ tubes, and then $2.5 \mu \mathrm{L}$ of $1.0 \mathrm{M} \mathrm{NaOH}$ and $15 \mathrm{ng}$ of diphenylamine (internal standard) in methanol (final concentration before mixing, approx. 35\%) was added. Chloroform $(60 \mu \mathrm{L})$ was added, and the mixture was shaken for $30 \mathrm{~min}$ at $2,000 \mathrm{rpm}$ and extracted by centrifugation at $22,000 \mathrm{~g}$ for $10 \mathrm{~min}$. The lipid layer was analyzed by GC-MS using a GCMS-QP2010 system (Shimadzu, Kyoto, Japan) with an AOC-5000 autoinjector (Shimadzu). The column was an Rtx-5 MS (internal diameter $30 \mathrm{~m} \times 0.25 \mathrm{~mm}$; film thickness $0.25 \mu \mathrm{m}$ ) (Shimadzu GLC, Tokyo, Japan). The pressure of the helium gas (carrier gas) was maintained at $100 \mathrm{kPa}$, and the volume of injected samples was $1-2 \mu \mathrm{L}$ with the instrument in the splitless mode. The temperature of the vaporizing chamber was $300^{\circ} \mathrm{C}$ and that of the column oven was raised in $25^{\circ} \mathrm{C} /$ min increments from 80 to $230^{\circ} \mathrm{C}$, then maintained at $230^{\circ} \mathrm{C}$ for $3.5 \mathrm{~min}$, followed by an increase to $320^{\circ} \mathrm{C}$ that was maintained for $6 \mathrm{~min}$. The interface temperature was $280^{\circ} \mathrm{C}$. Peaks were identified in SIM mode. Diphenylamine (internal standard) and cotinine were determined using peak areas for the mass numbers, 51.05 and 176.10, respectively. Peaks for mass numbers 169.1 and 98.1 were also monitored for confirmation. The coefficients of variation for six determinations of urine from non-smokers were $3-11 \%$ and the detection limit was $0.3 \mathrm{ng} / \mathrm{mL}$ (3 standard deviations, SD).

\section{HPLC analysis}

Urine from smokers was analyzed by HPLC with and without glucuronide deconjugation using $1 \mathrm{U} \beta$-glucuronidase (Sigma, Tokyo, Japan) $/ \mathrm{mL}$ urine at $37^{\circ} \mathrm{C}$ for $28 \mathrm{~h}$. Nicotine metabolites were extracted and determined as described by Zuccaro et al. [5]. Briefly, $500 \mu \mathrm{L}$ of urine and $200 \mu \mathrm{L} \mathrm{N}$-ethylnorcotinine $(10 \mu \mathrm{g} / \mathrm{mL})$ as an internal standard were mixed with $200 \mu \mathrm{L} 1.5 \mathrm{M} \mathrm{NaOH}$ and transferred to Extrelut-1 glass columns (Merck, Milan, Italy) that had been conditioned with $6 \mathrm{~mL}$ dichloromethane on the previous day. After $10 \mathrm{~min}$, the analytes were eluted under gravity with $5 \mathrm{~mL}$ of dichloromethane-isopropyl alcohol $(9: 1, \mathrm{v} / \mathrm{v})$. The organic phase was mixed with $100 \mu \mathrm{L}$ methanolic $\mathrm{HCl}(25 \mathrm{mM})$, evaporated to dryness under nitrogen, and dissolved in $200 \mu \mathrm{L}$ distilled water. Samples were eluted through a reversed-phase LC8DB column (internal diameter $5 \mu \mathrm{m}, 25 \mathrm{~cm} \times$ $4.6 \mathrm{~mm}$; Supelchem, Rome, Italy) using a mobile phase consisting of water-acetonitrile $(40: 1, \mathrm{v} / \mathrm{v})$ containing $5 \mathrm{~mL}$ triethylamine, $670 \mathrm{mg} / \mathrm{L}$ sodium heptane sulfonate, and $0.034 \mathrm{M}$ each of $\mathrm{K}_{2} \mathrm{HPO}_{4}$ and citric acid ( $\left.\mathrm{pH} 4.4\right)$, at a flow-rate of $1.6 \mathrm{~mL} / \mathrm{min}$ using the HPLC system, LC-10A (Shimadzu, Kyoto). The coefficients of variation for six determinations of urine from three non-smokers were $12-23 \%$ for cotinine and $13-19 \%$ for $3 \mathrm{HC}$. The detection limit $(3.3 \sigma / \mathrm{S})$ was $20 \mathrm{ng} / \mathrm{mL}$ for cotinine and $22 \mathrm{ng} / \mathrm{mL}$ for $3 \mathrm{HC}$ ( $\sigma$ residual SD, $S$ slope, $n=15$ within $0-100 \mathrm{ng} / \mathrm{mL}$ ).

The results were corrected with urinary creatinine levels that were measured using the LabAssay Creatinine kit (Wako Pure Chemical Industries, Tokyo, Japan).

\section{Results}

\section{Analytical validation of ELISA}

Characterization of the present ELISA was examined, and the detection limit was set to $1.3 \mathrm{ng} / \mathrm{mL}$ [Supplementary Electronic Material (ESM) Fig. 1 and Table 1]. The high cross-reactivity with $3 \mathrm{HC}$ and $3 \mathrm{HC}$-glucuronide (Table 2) indicated that IR-cotinine comprised a complex of the major nicotine metabolites, namely, cotinine, 3HC, and 3HC-glucuronide.

\section{Comparison of ELISA and GC-MS or HPLC}

We found significant correlations between IR-cotinine and both free and total cotinine; the regression curves of cotinine and IR-cotinine and of total cotinine and IR-cotinine are $y=0.31 x+1.27(r=0.91)$ and $y=0.73 x+2.44$ $(r=0.91)$, respectively (ESM Fig. 2). Significant but weaker correlations persisted when the creatinine-adjusted values were applied: $r=0.79$ between IR-cotinine and free cotinine $(y=0.31 x+1.20)$ and $r=0.86$ between IR-cotinine and total cotinine $(y=0.88 x+1.71)$.

The levels of cotinine were much higher determined by the ELISA than by GC-MS. In addition, IR-cotinine determined with the ELISA correlated more closely with the number of tobacco cigarettes smoked by the parents than did urinary cotinine levels measured using GC-MS (Fig. 1). The cotinine levels determined by ELISA also tended to be higher in the urine samples of those children whose mother was the only smoker in the family than in those of the children whose father was the only smoker (Fig. 2). Other smoking habits of parents also correlated 
Table 2 Comparisons of the various analytical methods of assaying cotinine

\begin{tabular}{|c|c|c|c|c|c|}
\hline Reference & Method $^{\mathrm{a}}$ & Antigen & Cross-reactivity $^{\mathrm{b}}(\%)$ & $\begin{array}{l}\text { Detection } \\
\text { limit }\end{array}$ & $\begin{array}{l}\text { Comparison with } \\
\text { chromatographic methods }\end{array}$ \\
\hline $\begin{array}{l}\text { Heinrich-Ramm et al. } \\
\text { [7] }\end{array}$ & RIA & & & & $\begin{array}{l}\text { 2.4- to } 2.9 \text {-fold higher than } \\
\text { GC-MS }\end{array}$ \\
\hline Kuo et al. [6] & ELISA & & & $0.39-0.46 \mathrm{ng} / \mathrm{mL}$ & $\begin{array}{l}\text { 0.6- to } 0.9 \text {-fold higher than } \\
\text { GC-nitrogen-phosphorus }\end{array}$ \\
\hline Ohta et al. [9] & ELISA & Cotinine-3-thyroglobulin & Nicotine (16) & $0.03 \mathrm{ng} / \mathrm{mL}$ & $\begin{array}{l}\text { 1.4-fold higher than } \\
\text { GC-MS }\end{array}$ \\
\hline Wielkoszynski et al. [8] & ELISA & 4-Carboxy- $\gamma$-cotinine & $\begin{array}{l}\text { 3HC (18.6), Nicotine } \\
\quad N \text {-oxide }(5.1)\end{array}$ & $3-3.5 \mathrm{ng} / \mathrm{mL}$ & 6-fold higher than TLC \\
\hline $\begin{array}{l}\text { Matsumoto et al. } \\
\text { (present study) }\end{array}$ & ELISA & Cotinine- $4-\gamma$-globulin & $\begin{array}{l}\mathrm{N}(0.2), \mathrm{N}-\mathrm{g}(0.0), \mathrm{C}-\mathrm{g} \\
(0.3), 3 \mathrm{HC}(101.3), \\
\text { 3HC-g (221.8) }\end{array}$ & $1.3 \mathrm{ng} / \mathrm{mL}$ & $\begin{array}{l}\text { 3.2- to } 5.9 \text {-fold higher than } \\
\text { GC-MS }\end{array}$ \\
\hline
\end{tabular}

with urinary cotinine levels measured by the ELISA, specifically IR-cotinine, more closely than the urinary cotinine levels measured using GC-MS (Fig. 2). The smoking habits of parents did not correlate with creatinine-adjusted cotinine levels; Spearman's rank correlation coefficients ranged from -0.17 to 0.15 ( $P$ values ranged from 0.10 to 0.89).

The correlation between IR-cotinine and total concentrations of the nicotine metabolites cotinine, 3HC, and 3HC-glucuronide in urine samples from 19 smokers was sufficient to confirm the cross-reactivity test described above $(y=0.99 x, r=0.94)$ (ESM Fig. 2).

\section{Discussion}

The results of this study validate a practical ELISA method for biologically monitoring exposure to tobacco smoke. Levels of IR-cotinine correlated with those of cotinine measured by the internationally validated GC-MS procedure. We also confirmed the relevance of the ELISA from the relationship between urinary IR-cotinine levels in kindergarten children and the smoking habits of their parents. However, ELISAs using other antibodies would require further validation because the cross-reactivity may differ. Data obtained with immunoassay systems should be handled carefully because cross-reactivity for each immunoassay systems differs (Table 2).

The major nicotine metabolites, namely, cotinine, 3HC, and 3HC-glucuronide, were reactive in the validated ELISA. About half of the nicotine taken up by humans is excreted into the urine in the form of these three metabolites (13, 34, and 7\%, respectively) [10]. Although measuring $3 \mathrm{HC}$ would be advantageous, the analysis of
3HC using GC-MS or HPLC is complicated, and the detection limit is too high; even solid phase extraction results in a detection limit of about $20 \mathrm{ng} / \mathrm{mL}$. In contrast, the detection limit of the ELISA for IR-cotinine is $1.3 \mathrm{ng} / \mathrm{mL}$, which is an important advantage, especially for monitoring passive smoking. Moreover, one of the advantages of our ELISA assay is that the properties of cross-reactivity have been well evaluated, so that the levels of nicotine metabolites are comparable to some degree with the levels detected using GC and LC. A number of earlier reports on ELISA systems provide relatively inadequate information in that large differences sometimes exist between IR levels and chromatographic levels and there is insufficient data on cross-reactivity (Table 2). Therefore, it can occasionally be difficult even to identify which compound the IR assay detects. This uncertainty makes any subsequent risk assessment difficult as these are often conducted using previously reported levels and internationally accepted cotinine levels based on GC and LC.

The antibody may cross-react with compounds other than the five nicotine metabolites that we evaluated, but according to the results from the urine of 19 smokers, the main compounds that cross-reacted with the cotinine antibody were the three nicotine metabolites described above.

Kitagawa et al. [11] found that about $4 \%$ of Japanese are homozygous for deletion-type alleles $(C Y P 2 A 6 * 4 / * 4)$ and have no CYP2A6 activity. Thus, one limitation of our ELISA is that values would be skewed in individuals with little or no CYP2A6 activity due to CYP2A6 polymorphisms, as these individuals would be partially or totally unable to convert nicotine to cotinine. Such individuals who are smokers excrete mainly nicotine and its 

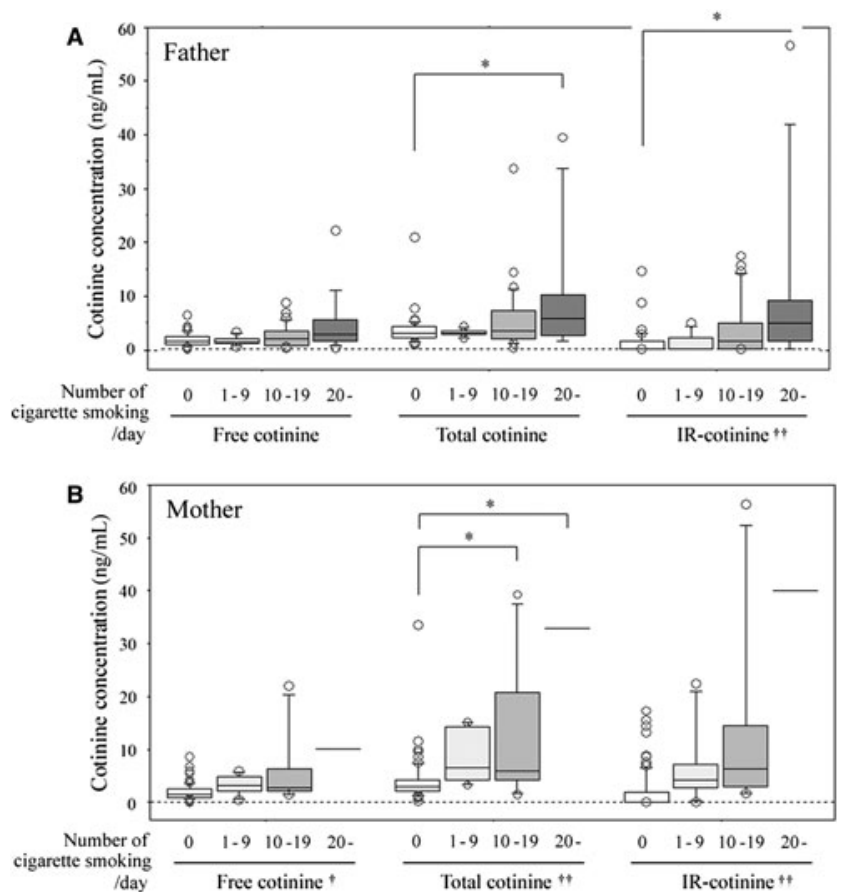

Fig. 1 Urine from 93 kindergarten children analyzed by enzymelinked immunosorbent assay (ELISA) and by chromatography-mass spectrometry (GC-MS). a Free and total cotinine levels determined by GC-MS and immunoreactive (IR)-cotinine levels in the urine of kindergarten children according to the amount of tobacco smoked by their father $(0,1-9,10-19$ and 20 cigarettes per day; $n=39,8,28$ and 14 , respectively). $* P<0.05$ by Scheffe's test after analysis of variance (ANOVA) using logarithmic analysis. ${ }^{\dagger} P<0.01$, by Kruskal-Wallis test. Spearman's rank correlation coefficients are $0.20(P=0.06), 0.26(P=0.02)$, and $0.41(P<0.01)$ for free cotinine, total cotinine and IR-cotinine, respectively. $\mathbf{b}$ Free and total cotinine levels determined by GC-MS and IR-cotinine levels in the urine of kindergarten children according to the amount of tobacco smoked by their mother $(0,1-9,10-19$ and 20 cigarettes per day; $n=79,6,6,1$, respectively). ${ }^{*} P<0.05$ by Scheffe's test after ANOVA using logarithmic analysis, ${ }^{\dagger} P<0.05,{ }^{\dagger \dagger} P<0.01$, by the Kruskal-Wallis test. Spearman's rank correlation coefficients are 0.33 $(P=0.002), 0.37 \quad(P<0.001)$, and $0.47 \quad(P<0.001)$ for free cotinine, total cotinine and IR-cotinine, respectively

metabolites nicotine glucuronide and nicotine $1^{\prime}-N$-oxide [12]. Another limitation of our ELISA is that IR-cotinine is difficult to compare with the cotinine analyzed by chromatographic methods. Nevertheless, many important results generated using GC or LC have already been reported. Analyses using ELISA should proceed with caution and always take these issues into consideration.

We found no significant correlation between creatinineadjusted cotinine values in the urine from kindergarten children and the number of cigarettes smoked by their parents, regardless of the good correlations between unadjusted values. Therefore, our results suggest that adjusting urinary cotinine values according to the creatinine concentration is possibly ineffective using kindergarten's urine as other studies have also shown that creatinine-adjusted


Fig. 2 Urine from 93 kindergarten children analyzed by ELISA and GC-MS. a Free and total cotinine levels determined by GC-MS and IR-cotinine levels in the urine of kindergarten children according to smoking status of parents: $a$ parents are non-smokers $(n=36)$, $b$ father smokes $(n=44), c$ mother smokes $(n=3), d$ both parents smoke $(n=10)$. $* P<0.05, * * P<0.01$ by Scheffe's test after ANOVA using logarithmic analysis, ${ }^{\dagger} P<0.01$ by the KruskalWallis test. Spearman's rank correlation coefficients are 0.28 $(P<0.01), 0.37(P<0.01)$, and $0.49(P<0.01)$ for free cotinine, total cotinine, and IR-cotinine, respectively. b Free and total cotinine levels determined by GC-MS and IR-cotinine levels in urine of kindergarten children according to smoking habits of the parents in the presence of their children: $a$ parents are non-smokers $(n=36)$, $b$ parents do not smoke in the presence of their children $(n=37)$, $c$ father or mother smokes in the presence of their children $(n=17)$, $d$ both parents smoke in the presence of their children $(n=3)$. ${ }^{\dagger} P<0.01$, Kruskal-Wallis test. Spearman's rank correlation coefficients are $0.14(P=0.17), 0.16(P=0.13)$ and $0.34(P<0.01)$ for free cotinine, total cotinine, and IR-cotinine, respectively

cotinine values based on children's urine samples are not meaningful $[13,14]$.

\section{Conclusion}

The results of our study suggest that this ELISA is useful for monitoring passive exposure to tobacco smoke; however, careful interpretation of the data is required.

Acknowledgments This study was funded by a grant from the Japanese Ministry of Health, Labour and Welfare (Clinical Cancer Research Project). The authors are grateful to Ms. Ranko Nishi and Mr. Tatsuya Takahashi for excellent technical assistance.

Conflict of interest statement The authors declare no conflict of interest. 


\section{References}

1. Jacob P 3rd, Shulgin AT, Yu L, Benowitz NL. Determination of the nicotine metabolite trans- $3^{\prime}$-hydroxycotinine in urine of smokers using gas chromatography with nitrogen-selective detection or selected ion monitoring. J Chromatogr B Anal Technol Biomed Life Sci. 1992;583:145-54.

2. Malaiyandi V, Goodz SD, Sellers EM, Tyndale RF. CYP2A6 genotype, phenotype, and the use of nicotine metabolites as biomarkers during ad libitum smoking. Cancer Epidemiol Biomarkers Prev. 2006;15:1812-9.

3. Services USDoHaH: The health consequences of involuntary exposure to tobacco smoke. In: A report of the Surgeon General. U.S. Department of Health and Human Services, Atlanta, GA; 2006.

4. Man CN, Gam LH, Ismail S, Lajis R, Awang R. Simple, rapid and sensitive assay method for simultaneous quantification of urinary nicotine and cotinine using gas chromatography-mass spectrometry. J Chromatogr B Anal Technol Biomed Life Sci. 2006;844:322-7.

5. Zuccaro P, Altieri I, Rosa M, Passa AR, Pichini S, Pacifici R. Solid-phase extraction of nicotine and its metabolites for highperformance liquid chromatographic determination in urine. J Chromatogr B Biomed Appl. 1995;668:187-8.

6. Kuo HW, Yang JS, Chiu MC. Determination of urinary and salivary cotinine using gas and liquid chromatography and enzyme-linked immunosorbent assay. J Chromatogr B Anal Technol Biomed Life Sci. 2002;768:297-303.

7. Heinrich-Ramm R, Wegner R, Garde AH, Baur X. Cotinine excretion (tobacco smoke biomarker) of smokers and non- smokers: comparison of GC/MS and RIA results. Int J Hyg Environ Health. 2002;205:493-9.

8. Wielkoszynski T, Tyrpien K, Szumska M. The enzyme-linked immunosorbent assay (ELISA) method for nicotine metabolites determination in biological fluids. J Pharm Biomed Anal. 2009; 49:1256-60.

9. Ohta M, Kunihiro T, Geta Y, Kojima Y, Fujimani A, Hanada S, Sakuraoka M, Ino T, Ohta K. Development of high-sensitive EIA for cotinine, a biomarker of exposure to passive smoking, and its clinical applications (in Japanese). Jpn J Med Pharmaceut Sci. 2007;58:589.

10. Benowitz NL, Jacob P 3rd, Fong I, Gupta S. Nicotine metabolic profile in man: comparison of cigarette smoking and transdermal nicotine. J Pharmacol Exp Ther. 1994;268:296-303.

11. Kitagawa K, Kunugita N, Katoh T, Yang M, Kawamoto T. The significance of the homozygous CYP2A6 deletion on nicotine metabolism: a new genotyping method of CYP2A6 using a single PCR-RFLP. Biochem Biophys Res Commun. 1999;262:146-51.

12. Yamanaka H, Nakajima M, Nishimura K, Yoshida R, Fukami T, Katoh M, et al. Metabolic profile of nicotine in subjects whose CYP2A6 gene is deleted. Eur J Pharmaceut Sci Off J Eur Fed Pharmaceut Sci. 2004;22:419-25.

13. Fried PA, Perkins SL, Watkinson B, McCartney JS. Association between creatinine-adjusted and unadjusted urine cotinine values in children and the mother's report of exposure to environmental tobacco smoke. Clin Biochem. 1995;28:415-20.

14. Dempsey D, Jacob P 3rd, Benowitz NL. Nicotine metabolism and elimination kinetics in newborns. Clin Pharmacol Ther. 2000; 67:458-65. 